\title{
Addendum
}

\section{A Stochastic Particle System Modeling the Carleman Equation ${ }^{1}$}

\author{
S. Caprino, A. DeMasi, E. Presutti, and M. Pulvirenti
}

Received November 21, 1989

This addendum concerns the statement after (3.19), which says that the hierarchy of equations (3.11) has a unique solution [in (3.11) there should be a minus sign in front of $\sigma \Delta$ ].

Such a statement is easily proven when one has an a priori bound on the $j$-body correlation functions of the form $c^{j}$, with some fixed $c$ which does not depend on $j$. Our a priori bound, however, grows like $e^{c j^{2}}$ and the proof of uniqueness becomes much more delicate: uniqueness might not even be true, in general, in the class of correlation functions bounded only by $e^{c j^{2}}$. Our proof, as we are going to see, exploits in an essential way the presence of the heat kernel, $\sigma \neq 0$, in the same way used to prove the $a$ priori bound.

We write in integral form the hierarchy of equations (3.11) for the correlation functions $h_{j}^{\sigma}$ and we get

$$
h_{j}^{\sigma}(\cdot, t)=V_{j, t} f_{j, 0}+\int_{0}^{t} d s V_{j, t-s} C_{j, j+1} h_{j+1}^{\sigma}(\cdot, s)
$$

where $f_{j, 0}$ are the correlation functions at time 0 , namely

$$
f_{\mathrm{j}, 0}=\prod_{k=1}^{j} f\left(x_{k}, v_{k}, 0\right)
$$

$f(x, v, 0)$ being the initial datum for the Carleman equation, assumed to be

${ }^{1}$ This paper appeared in J. Stat. Phys. 55:625-638 (1989). 
a normalized, nonnegative function in $\mathbb{C}^{0}\left([0,1]^{2}\right)$. The collision operator $C_{j, j+1}$ is given by

$$
\begin{gathered}
C_{j, j+1}=\sum_{i=1}^{j} \sum_{b_{i}= \pm} C_{j, j+1}^{i, b_{l}} \\
C_{j, j+1}^{i, \pm} g_{j+1}\left(x_{1}, v_{1}, \ldots, x_{j}, v_{j}\right) \\
=\mp g_{j+1}\left(x_{1}, v_{1}, \ldots, x_{i}, \pm v_{i}, \ldots, x_{j}, v_{j}, x_{i}, \pm v_{i}\right)
\end{gathered}
$$

Iterating (1) $n$ times, we get

$$
\begin{aligned}
h_{j}^{\sigma}(\cdot, t)= & V_{j, t} f_{j, 0} \\
& +\sum_{m=1}^{n-1} \int_{0}^{t} d s_{1} \cdots \int_{0}^{s_{m-1}} d s_{m} \sum_{i_{1}, \ldots, i_{m}} \sum_{b_{1}, \ldots, b_{m}} V_{j, t-s_{1}} C_{j, j+1}^{i_{1}, b_{1}} \cdots \\
& \times V_{j+m-1, s_{m-1}-s_{m}} C_{j+m-1, j+m}^{i_{m}, b_{m}} V_{j+m, s_{m}} f_{j+m, 0} \\
& +\int_{0}^{t} d s_{1} \cdots \int_{0}^{s_{n-1}} d s_{n} \sum_{i_{1}, \ldots, i_{n}} \sum_{b_{1}, \ldots, b_{n}} V_{j, t-s_{1}} C_{j, j+1}^{i_{1}, b_{1}} \cdots \\
& \times V_{j+n-1, s_{n-1}-s_{n}} C_{j+n-1, j+n}^{i_{n}, b_{n}}, h_{j+n}^{\sigma}\left(\cdot, s_{n}\right)
\end{aligned}
$$

We bound the first two terms on the right-hand side of (4) by

$$
\sum_{m=0}^{n-1} \frac{(j+m) !}{j ! m !} 2^{m} t^{m} c^{j+m}
$$

where $c \equiv \max f(x, v, 0)$. As we shall see at the end of this addendum, we can also prove a bound which is independent of the sup-norm of the initial datum.

For $t$ small enough, precisely for $2 t c<1,(5)$ is bounded uniformly on $n$. The problem is therefore to control the remainder in (4). Let us fix the values $n \geqslant 2, s_{1}, \ldots, s_{n}, i_{1}, \ldots, i_{n}$, and $b_{1}, \ldots, b_{n}$. Call

$$
A \equiv\left|C_{j+n-2, j+n-1}^{i_{n-1}, b_{n-1}} V_{j+n-1, s_{n-1}-s_{n}} C_{j+n-1, j+n}^{l_{n}, b_{n}} h_{j+n}^{\sigma}\left(\cdot, s_{n}\right)\right|
$$

Assume first that the label $j+n-1 \neq i_{n}$; then, by integrating over the $(j+n-1)$ th particle and using (3.3b) [see (3.2) for notation] and (3.8), we get

$$
A \leqslant c_{\sigma}\left(s_{n-1}-s_{n}\right)^{-1 / 2}\left|V_{j+n-2, s_{n-1}-s_{n}} C_{j+n-2, j+n-1}^{i_{n}, b_{n}} h_{j+n-1}^{\sigma}\left(\cdot, s_{n}\right)\right|
$$

If, on the other hand, $i_{n}=j+n-1$, we can use the symmetry of $A$ under the exchange of $j+n-1$ and $i_{n-1}$ due to the fact that the particles with labels $j+n-1$ and $i_{n-1}$ are in the same state at time $s_{n-1}$. 
We can now use the semigroup property and iterate the procedure. We therefore have that the last integral in (4) is bounded, in absolute value, by

$\frac{(j+n) !}{j !} 2^{n} c_{\sigma}^{n-1}\left\|h_{j+1}^{\sigma}\right\|_{\infty} \int_{0}^{t} d s_{1} \cdots \int_{0}^{s_{n-1}} d s_{n}\left(s_{1}-s_{n}\right)^{-1 / 2} \cdots\left(s_{n-1}-s_{n}\right)^{-1 / 2}$

where $\left\|h_{j+1}^{\sigma}\right\|_{\infty}$ is the sup over space and over times $s \leqslant(2 c)^{-1}, c$ being as in (5): we consider for the moment only times $t$ which are smaller than $(2 c)^{-1}$. The expression in (8) is equal to

$$
\frac{(j+n) !}{j !} 2^{n} c_{\sigma}^{n}\left\|h_{j+1}^{\sigma}\right\|_{\infty} \frac{2^{n+1}}{(n-1) !(n+1)} t^{(n+1) / 2}
$$

For $4 c_{\sigma} t^{1 / 2}<1$ this term vanishes when $n \rightarrow \infty$. Hence, for $t<\min \left\{\left(4 c_{\sigma}\right)^{-2},(2 c)^{-1}\right\}$, [cf. (5)] $h_{j}^{\sigma}(\cdot, t)$ is given by the limit as $n \rightarrow \infty$ of the first two terms in (4). This proves that in the same time interval

$$
h_{j}^{\sigma}(\cdot, t)=\prod_{i=1}^{l} f(\cdot, t)
$$

where $f$ solves (2.17). By (3.18) we can start again and reach times twice as large as before. By iteration we then prove that (10) extends to all times.

A final remark: the same argument used to control the last integral in (4) allows one to prove a bound for the first sum in (4) which is independent of $\|f(\cdot, 0)\|_{\infty}$. 